J. Clin. Chem. Clin. Biochem.

Vol. 19, 1981, pp. 1137-1144

\title{
The Importance of a Blind Control in the Establishment of Assigned Values in Control Sera ${ }^{1}$ )
}

\section{The Establishment of Assigned Values in Control Sera, I.}

By H. Passing

Hoechst AG,POB 800 320, D-6230 Frankfurt (M) 80, Federal Republic of Germany,

\section{B. Müller}

Behringwerke AG, POB 1140,D-3550 Marburg 1, Federal Republic of Germany and

\section{H. Brettschneider}

Boehringer Mannheim GmbH, Nonnenwald, D-8122 Penzberg, Federal Republic of Germany

(Received February 23/July 15, 1981)

Summary: We describe a study by which the establishment of assigned values of a control serum was simulated. The study covered two controls: The internal known control and a blind control. Seven constituents were analyzed in 10 or 11 laboratories, respectively, yielding a total of 72 sets of analytical values. Each set covered double determinations within approximately 18 series for each sample.

The course of a blind control correlates better with the unknown sample for which assigned values are to be determined than does a known control. Out of 72 sets 5 sets were found incorrect. Out of these, 2 sets could be recognized exclusively by means of the blind control, and 2 others primarily by means of the known control; one of these could be detected by means of the double determinations. Consequently, a blind control has a greater control efficiency than double determinations.

Die Bedeutung einer Blindkontrolle bei der Ermittlung von Sollwerten in Kontrollseren Ermittlung von Sollwerten in Kontrollseren, $I$.

Zusammenfassung: Wir beschreiben eine Studie, mit deren Hilfe die Ermittlung von Sollwerten eines Kontrollserums simuliert wurde. Die Studie umfaßte zwei Kontrollen: die interne bekannte Kontrolle und eine Blindkontrolle. Sịeben Bestandteile wurden jeweils in 10 bis 11 Läboratorien analysiert, so daß sich insgesamt 72 Ergebnismengen ergaben. Jede Ergebnismenge umfaßte für jedes Serum Doppelbestimmungen in ungefähr 18 Serien.

Der Verlauf einer Blindkontrolle korreliert besser mit der unbekannten Probe, für die Sollwerte bestimmt werden sollen, als der Verlauf einer bekannten Kontrolle. Von den 72 Ergebnismengen wurden 5 als fehlerhaft erkannt. Von diesen konnten 2 allein mit Hilfe der Blindkontrolle und 2 weitere in erster Linie mit Hilfe der bekannten Kontrolle erkannt werden. Hiervon konnte eine mit Hilfe der Doppelbestimmungen entdeckt werden. Eine Blindkontrolle besitżt also größere Kontrolleffizienz als Doppelbestimmungen.

\section{Introduction}

There are various designs for the establishment of assigned values in control sera $(2,3,4)$. They vary considerably with respect to the number of laboratories, and the number and the arrangement of determinations

1) A preliminary report is given in 1.c. (1) within each laboratory. Roughly speaking, either few laboratories but many determinations are demanded or inversely more or fewer laboratories with few determinations are required. Sometimes the arrangement of the analyses within the laboratories is even prescribed as a double determination. Furthermore, the statistical methods for the calculation of the assigned value and its uncertainty interval differ essentially. 
Unfortunately, arguments based on experimental data for these designs and evaluations have not been reported. No systematic comparisons between these different methods and their implications on the assigned value and its uncertainty interval have been published until now.

Therefore it was our intention to investigate the influence of different designs and statistical methods on the assigned value and uncertainty interval, in order to elaborate a model which gives accurate assigned values with acceptable expenditure with a high degree of security.

It has become standard in the determination of most blood constituents to monitor the results by appropriate control materials. On the other hand it is known that the results of these controls do not always represent the correct degree of precision and accuracy in the clinical laboratory, because controls are handled with special care. Moreover, previous results from the same control and the same experiment influence the technician in reading subsequent values $(5,6)$. Furthermore, the uncertainty interval of the different controls used by different laboratories may have been established under different conditions and for different purposes yielding systematically smaller or broader intervals. Consequently, the participating reference laboratories use different procedures to monitor their results. Finally, different serum-specific problems can arise from different control sera so that e.g. the values of the control serum are all correct, whereas the values of the unknown sample are all systematically wrong. This would mean that the control is completely ineffective.

A common control serum was distributed to all participants in order to check the performance of the laboratories uniformly. The common control served as a blind control, i.e. its assigned values and uncertainty intervals were known to the project leader only. This paper describes the errors which were detected by different control procedures.

In the further papers of this series various statistical methods are examined with respect to their adequacy. Different designs for the establishment of assigned values are compared critically. This will finally lead to an optimized design and evaluation procedure.

\section{Materials and Methods}

The 12 laboratories participating in this study are members of the Verband der Diagnostica- und Diagnosticageräte-Hersteller (VDGH, Association of Diagnostics and Diagnostics Instrumentation Manufacturers).

(Monitrol II (Dade), lot no. $58 \mathrm{~B}$, served as the unknown sample for the establishment of assigned values and their uncertainty intervals.

The common in-house controls were used as known controls, namely ${ }^{\circledR}$ Normosic and PKR (Asid Bonz und Sohn GmbH), @Kontrollogen L (Behringwerke AG), ®Serodos (Boehringer
Ingelheim Diagnostika GmbH), ${ }^{\circledR}$ Precinorm $U$ and $E$ and @Precipath $\mathrm{E}$ and $\mathrm{S}$ (Boehringer Mannheim $\mathrm{GmbH}$ ), and Validate A (Goedecke AG).

Precinorm U (Boehringer Mannheim GmbH), lot. no. 618, was used as a blind control.

The study was based on the following constituents and methods: Creatinine, Jaffé reaction without deproteinization $(7,8,9)$, reading at $\mathrm{Hg} 492 \mathrm{~nm}$,

glucose, enzymatic with hexokinase/glucose 6-phosphate dehydrogenase $(10,11,12)$, reading at $\mathrm{Hg} 365 \mathrm{~nm}$,

urea, urease and subsequent Berthelot reaction $(13,14)$, reading at $\mathrm{Hg} 546 \mathrm{~nm}$,

alanine aminotransferase ${ }^{2}$ ), standard method of Deutsche Gesellschaft für Klinische Chemie (15),

aspartate aminotransferase ${ }^{2}$ ), standard method of Deutsche Gesellschaft für Klinische Chemie (15),

creatine kinase $\left.{ }^{2}\right), \mathrm{N}$-acetyl cysteine activated $(16,17)$, $\gamma$-glutamyltransferase ${ }^{2}$ ), substrate $\boldsymbol{\gamma}$-glutamyl 3-carboxy 4-nitroanilide (18).

All enzyme activities were measured at $25^{\circ} \mathrm{C}$ and $\mathrm{Hg} 365 \mathrm{~nm}$ except for $\gamma$-glutamyltransferase which was read at $\mathrm{Hg} 405 \mathrm{~nm}$.

The following factors were kept constant in order to avoid systematic bias due to modifications in the performance of the analyses: the same type of photometer (EPPENDORF Gerätebau) was used; the reagents for each constituent were delivered from one manufacturer and derived from one common lot for all participants; the analyses were performed at final volumes greater than $2.0 \mathrm{ml}$ according to the package inserts.

Table 1 shows the number of participating laboratories for each constituent.

Tab. 1. Number of laboratories participating in the study and remaining after eliminations.

\begin{tabular}{lll}
\hline Constituent & $\begin{array}{l}\text { Number of } \\
\text { laboratories } \\
\text { participatinga) }\end{array}$ & $\begin{array}{l}\text { Number of } \\
\text { laboratories } \\
\text { remaining }\end{array}$ \\
\hline Creatinine & 10 & 9 \\
Glucose & 10 & 9 \\
Urea & 10 & 9 \\
$\begin{array}{l}\text { Alanine amino- } \\
\text { transferase }\end{array}$ & 11 & 11 \\
$\begin{array}{l}\text { Aspartate amino- } \\
\text { transferase }\end{array}$ & 11 & 11 \\
$\begin{array}{l}\text { Creatine kinase } \\
\text { r-Glutamyltransferase }\end{array}$ & 10 & 9 \\
\hline Total & 72 & 9 \\
\hline
\end{tabular}

a) No constituent was determined by all 12 laboratories participating in this study.

Double determinations were performed within approximately 18 independent series, for each of the three sera and in each laboratory. Table 2 shows mean, standard deviation, median, and the smallest and the largest analytical value of the series, separated according to the first and to the second determination of the double determinations of the unknown sample, and the standard deviation within series.

We simulated single determinations by using the first values only of the double determinations.

\footnotetext{
2) Enzymes: Glutamate-pyruvate-transaminase alanine aminotransferase $=L$-alanine: 2 -oxoglutarate aminotransferase EC 2.6.1:2; glutamate-oxalacetate-transaminase = aspartate aminotransferase $=L$-aspartate: 2 -oxoglu tarate aminotransferase EC 2.6.1.1; $\gamma$-glutamyl transferase = (5-glutamyl)peptide: aminoacid 5-glutamyltransférase EC 2.3.2.2; creatine kinase $=$ ATP: creatine $\mathrm{N}$-phosphotransferase EC 2.7.3.2.
} 
Tab. 2. Survey of the original dataa) of the unkown sample.

\begin{tabular}{|c|c|c|c|c|c|c|c|c|c|c|c|c|}
\hline \multirow{3}{*}{$\begin{array}{l}\begin{array}{l}\text { Consti- } \\
\text { tuent }\end{array} \\
\begin{array}{l}\text { Creatinine } \\
(\mu \mathrm{mol} / \mathrm{l})\end{array}\end{array}$} & \multirow[b]{2}{*}{$\begin{array}{l}\text { Labor- } \\
\text { atoryb) }\end{array}$} & \multicolumn{5}{|c|}{ First values } & \multicolumn{6}{|c|}{ Second values } \\
\hline & & Mean & SDC) & Mediand) & Min.e) & Max.f) & Mean & $\mathrm{SDC})$ & Mediand) & Min.e) & $\operatorname{Max} . f$ & $\begin{array}{l}\text { SD } \\
\text { within } \\
\text { series }\end{array}$ \\
\hline & $\begin{array}{l}\text { A } \\
\text { C } \\
\text { D } \\
\text { E } \\
\text { F } \\
\text { G } \\
\text { H } \\
\text { I } \\
\text { J } \\
\text { K }\end{array}$ & $\begin{array}{l}301 \\
318 \\
306 \\
373 \\
325 \\
319 \\
311 \\
311 \\
302 \\
290\end{array}$ & $\begin{array}{r}16 \\
12 \\
8 \\
12 \\
11 \\
8 \\
9 \\
2 \\
9 \\
9\end{array}$ & $\begin{array}{l}307 \\
319 \\
307 \\
375 \\
325 \\
321 \\
308 \\
311 \\
303 \\
290\end{array}$ & $\begin{array}{l}265 \\
301 \\
288 \\
348 \\
306 \\
305 \\
298 \\
308 \\
280 \\
270\end{array}$ & $\begin{array}{l}321 \\
342 \\
321 \\
392 \\
346 \\
331 \\
326 \\
315 \\
315 \\
308\end{array}$ & $\begin{array}{l}301 \\
322 \\
305 \\
382 \\
322 \\
318 \\
308 \\
312 \\
300 \\
286\end{array}$ & $\begin{array}{r}17 \\
14 \\
10 \\
11 \\
12 \\
8 \\
8 \\
3 \\
9 \\
9\end{array}$ & $\begin{array}{l}303 \\
321 \\
307 \\
385 \\
322 \\
320 \\
306 \\
312 \\
300 \\
286\end{array}$ & $\begin{array}{l}265 \\
298 \\
286 \\
354 \\
303 \\
304 \\
297 \\
307 \\
276 \\
271\end{array}$ & $\begin{array}{l}331 \\
346 \\
321 \\
397 \\
341 \\
330 \\
324 \\
316 \\
311 \\
303\end{array}$ & $\begin{array}{l}3 \\
7 \\
5 \\
7 \\
3 \\
3 \\
2 \\
2 \\
5 \\
5\end{array}$ \\
\hline $\begin{array}{l}\text { Glucose } \\
(\mathrm{mmol} / \mathrm{l})\end{array}$ & $\begin{array}{l}\text { A } \\
\text { C } \\
\text { D } \\
\text { E } \\
\text { F } \\
\text { G } \\
\text { H } \\
\text { I } \\
\text { J } \\
\text { K }\end{array}$ & $\begin{array}{l}12.0 \\
11.6 \\
12.5 \\
11.8 \\
12.0 \\
12.5 \\
12.3 \\
12.1 \\
11.9 \\
12.5\end{array}$ & $\begin{array}{l}0.4 \\
0.2 \\
0.2 \\
0.2 \\
0.5 \\
0.2 \\
0.8 \\
0.2 \\
0.3 \\
0.4\end{array}$ & $\begin{array}{l}11.9 \\
11.6 \\
12.5 \\
11.8 \\
12.1 \\
12.5 \\
12.2 \\
12.1 \\
11.9 \\
12.5\end{array}$ & $\begin{array}{l}11.5 \\
11.2 \\
12.0 \\
11.4 \\
11.1 \\
12.0 \\
11.6 \\
11.9 \\
11.4 \\
11.7\end{array}$ & $\begin{array}{l}12.7 \\
11.9 \\
12.9 \\
12.1 \\
12.6 \\
12.9 \\
14.9 \\
12.5 \\
12.5 \\
12.9\end{array}$ & $\begin{array}{l}12.1 \\
11.7 \\
12.5 \\
11.8 \\
12.1 \\
12.5 \\
12.2 \\
12.1 \\
11.9 \\
12.6\end{array}$ & $\begin{array}{l}0.5 \\
0.3 \\
0.2 \\
0.2 \\
0.4 \\
0.2 \\
0.5 \\
0.2 \\
0.3 \\
0.2\end{array}$ & $\begin{array}{l}11.9 \\
11.8 \\
12.5 \\
11.8 \\
12.2 \\
12.5 \\
12.1 \\
12.1 \\
11.9 \\
12.7\end{array}$ & $\begin{array}{l}11.6 \\
11.2 \\
12.2 \\
11.5 \\
11.3 \\
12.2 \\
11.5 \\
11.9 \\
11.4 \\
12.2\end{array}$ & $\begin{array}{l}13.2 \\
12.1 \\
13.1 \\
12.1 \\
12.7 \\
13.0 \\
13.5 \\
12.3 \\
12.7 \\
12.9\end{array}$ & $\begin{array}{l}0.2 \\
0.2 \\
0.1 \\
0.1 \\
0.3 \\
0.2 \\
0.4 \\
0.2 \\
0.1 \\
0.2\end{array}$ \\
\hline $\begin{array}{l}\text { Urea } \\
(\mathrm{mmol} / \mathrm{l})\end{array}$ & $\begin{array}{l}\text { A } \\
\text { C } \\
\text { D } \\
\text { E } \\
\text { F } \\
\text { G } \\
\text { H } \\
\text { I } \\
\text { J } \\
\text { K }\end{array}$ & $\begin{array}{l}12.6 \\
13.4 \\
12.8 \\
12.7 \\
13.3 \\
13.4 \\
12.2 \\
13.4 \\
13.0 \\
13.0\end{array}$ & $\begin{array}{l}0.3 \\
0.3 \\
0.4 \\
0.5 \\
0.3 \\
0.3 \\
1.2 \\
0.2 \\
0.3 \\
0.2\end{array}$ & $\begin{array}{l}12.6 \\
13.5 \\
12.8 \\
12.8 \\
13.4 \\
13.4 \\
12.4 \\
13.4 \\
13.1 \\
13.0\end{array}$ & $\begin{array}{l}12.1 \\
12.9 \\
12.0 \\
11.4 \\
12.8 \\
13.0 \\
10.1 \\
12.9 \\
12.4 \\
12.6\end{array}$ & $\begin{array}{l}13.1 \\
13.8 \\
13.3 \\
13.6 \\
13.7 \\
13.8 \\
14.8 \\
13.8 \\
13.4 \\
13.3\end{array}$ & $\begin{array}{l}12.7 \\
13.3 \\
12.8 \\
12.7 \\
13.3 \\
13.4 \\
12.3 \\
13.3 \\
12.9 \\
12.9\end{array}$ & $\begin{array}{l}0.4 \\
0.3 \\
0.3 \\
0.5 \\
0.8 \\
0.3 \\
1.7 \\
0.3 \\
0.2 \\
0.3\end{array}$ & $\begin{array}{l}12.8 \\
13.4 \\
12.8 \\
12.8 \\
13.4 \\
13.4 \\
12.5 \\
13.3 \\
12.9 \\
12.9\end{array}$ & $\begin{array}{r}12.1 \\
12.8 \\
12.0 \\
11.5 \\
11.2 \\
13.0 \\
9.5 \\
12.9 \\
12.5 \\
12.5\end{array}$ & $\begin{array}{l}13.5 \\
13.8 \\
13.2 \\
13.5 \\
14.2 \\
14.0 \\
15.6 \\
13.9 \\
13.2 \\
13.3\end{array}$ & $\begin{array}{l}0.3 \\
0.2 \\
0.1 \\
0.3 \\
0.6 \\
0.2 \\
1.2 \\
0.2 \\
0.2 \\
0.1\end{array}$ \\
\hline $\begin{array}{l}\text { Alanine } \\
\text { amino- } \\
\text { transferase } \\
\text { (U/1) }\end{array}$ & $\begin{array}{l}\mathbf{A} \\
\mathbf{B} \\
\mathbf{C} \\
\mathbf{D} \\
\mathbf{E} \\
\mathbf{F} \\
\mathbf{G} \\
\mathbf{H} \\
\mathbf{I} \\
\mathbf{J} \\
\mathbf{K}\end{array}$ & $\begin{array}{l}64.0 \\
66.3 \\
62.6 \\
62.8 \\
62.0 \\
62.7 \\
65.8 \\
62.2 \\
63.2 \\
59.7 \\
62.5\end{array}$ & $\begin{array}{l}2.1 \\
2.0 \\
1.9 \\
1.5 \\
1.7 \\
1.5 \\
1.1 \\
1.5 \\
0.7 \\
1.3 \\
1.7\end{array}$ & $\begin{array}{l}64.7 \\
66.9 \\
62.9 \\
62.6 \\
61.8 \\
63.1 \\
66.2 \\
63.1 \\
63.3 \\
59.5 \\
63.2\end{array}$ & $\begin{array}{l}60.2 \\
63.1 \\
58.5 \\
60.5 \\
59.9 \\
59.3 \\
64.2 \\
59.3 \\
62.3 \\
57.4 \\
59.5\end{array}$ & $\begin{array}{l}67.0 \\
70.7 \\
66.3 \\
65.0 \\
66.9 \\
65.0 \\
67.3 \\
65.0 \\
64.4 \\
61.7 \\
64.9\end{array}$ & $\begin{array}{l}63.4 \\
65.5 \\
63.4 \\
62.2 \\
62.3 \\
62.4 \\
65.6 \\
62.5 \\
63.5 \\
59.5 \\
62.6\end{array}$ & $\begin{array}{l}1.7 \\
1.5 \\
2.2 \\
1.8 \\
1.1 \\
1.2 \\
1.3 \\
3.1 \\
0.7 \\
1.1 \\
2.2\end{array}$ & $\begin{array}{l}63.0 \\
65.0 \\
63.0 \\
61.8 \\
62.5 \\
62.5 \\
65.8 \\
63.1 \\
63.4 \\
59.7 \\
62.2\end{array}$ & $\begin{array}{l}61.0 \\
63.1 \\
60.7 \\
57.8 \\
59.9 \\
60.6 \\
63.1 \\
55.4 \\
62.3 \\
57.7 \\
59.1\end{array}$ & $\begin{array}{l}67.4 \\
68.8 \\
67.6 \\
64.5 \\
64.4 \\
65.0 \\
67.5 \\
68.8 \\
64.8 \\
61.2 \\
66.2\end{array}$ & $\begin{array}{l}1.4 \\
1.5 \\
1.1 \\
1.6 \\
1.2 \\
0.8 \\
0.4 \\
1.8 \\
0.7 \\
1.0 \\
1.0\end{array}$ \\
\hline $\begin{array}{l}\text { Aspartate } \\
\text { amino- } \\
\text { transferase } \\
(\mathrm{U} / \mathrm{l}) \\
.\end{array}$ & $\begin{array}{l}\text { A } \\
\text { B } \\
\text { C } \\
\text { E } \\
\text { F } \\
\text { G } \\
H \\
\dot{I} \\
\text { J } \\
\text { K } \\
\text { L }\end{array}$ & $\begin{array}{l}41.1 \\
40.2 \\
40.3 \\
40.4 \\
39.3 \\
40.6 \\
39.6 \\
39.1 \\
39.8 \\
39.8 \\
40.2\end{array}$ & $\begin{array}{l}1.9 \\
0.9 \\
1.7 \\
1.0 \\
1.9 \\
0.7 \\
1.4 \\
0.6 \\
1.2 \\
1.9 \\
1.2\end{array}$ & $\begin{array}{l}41.0 \\
40.2 \\
40.6 \\
40.4 \\
39.5 \\
40.6 \\
40.1 \\
39.2 \\
40.0 \\
39.2 \\
40.5\end{array}$ & $\begin{array}{l}38.5 \\
38.7 \\
37.1 \\
39.2 \\
32.5 \\
39.6 \\
38.2 \\
38.2 \\
37.7 \\
38.2 \\
36.7\end{array}$ & $\begin{array}{l}45.4 \\
41.6 \\
43.1 \\
42.5 \\
40.8 \\
41.8 \\
42.1 \\
40.2 \\
42.1 \\
45.4 \\
42.1\end{array}$ & $\begin{array}{l}40.2 \\
40.2 \\
40.2 \\
40.0 \\
39.3 \\
40.7 \\
39.4 \\
39.3 \\
39.7 \\
40.1 \\
40.4\end{array}$ & $\begin{array}{l}2.2 \\
1.0 \\
1.5 \\
0.9 \\
1.9 \\
0.7 \\
1.3 \\
0.6 \\
1.5 \\
2.2 \\
1.1\end{array}$ & $\begin{array}{l}39.3 \\
40.2 \\
40.7 \\
40.0 \\
39.9 \\
40.9 \\
40.1 \\
39.5 \\
39.7 \\
39.4 \\
40.5\end{array}$ & $\begin{array}{l}37.7 \\
38.7 \\
37.7 \\
38.7 \\
32.5 \\
39.3 \\
38.2 \\
38.2 \\
37.8 \\
36.2 \\
38.2\end{array}$ & $\begin{array}{l}44.5 \\
42.5 \\
42.6 \\
41.1 \\
41.4 \\
41.8 \\
42.1 \\
39.8 \\
43.5 \\
46.3 \\
42.8\end{array}$ & $\begin{array}{l}1.6 \\
0.3 \\
0.9 \\
0.5 \\
0.5 \\
0.4 \\
0.9 \\
0.6 \\
0.5 \\
1.0 \\
0.5\end{array}$ \\
\hline $\begin{array}{l}\text { Creatine } \\
\text { kinase } \\
\text { (U/1) }\end{array}$ & $\begin{array}{l}\mathbf{A} \\
\mathbf{C} \\
\mathbf{D} \\
\dot{\mathbf{E}} \\
\mathbf{F} \\
\mathbf{G} \\
\dot{H} \\
\mathbf{I} \\
\mathbf{J} \\
\dot{\mathbf{K}}\end{array}$ & $\begin{array}{l}225 \\
158 \\
189 \\
221 \\
221 \\
260 \\
232 \\
204 \\
249 \\
226\end{array}$ & $\begin{array}{r}18 \\
29 \\
28 \\
10 \\
17 \\
6 \\
16 \\
3 \\
11 \\
14\end{array}$ & $\begin{array}{l}224 \\
160 \\
191 \\
223 \\
223 \\
262 \\
230 \\
204 \\
248 \\
229\end{array}$ & $\begin{array}{l}196 \\
106 \\
119 \\
201 \\
191 \\
250 \\
201 \\
198 \\
227 \\
193\end{array}$ & $\begin{array}{l}257 \\
199 \\
228 \\
235 \\
243 \\
268 \\
253 \\
210 \\
272 \\
243\end{array}$ & $\begin{array}{l}223 \\
158 \\
191 \\
220 \\
222 \\
260 \\
225 \\
205 \\
249 \\
225\end{array}$ & $\begin{array}{r}15 \\
28 \\
30 \\
9 \\
16 \\
5 \\
14 \\
4 \\
10 \\
13\end{array}$ & $\begin{array}{l}226 \\
159 \\
197 \\
220 \\
221 \\
260 \\
223 \\
206 \\
248 \\
230\end{array}$ & $\begin{array}{l}200 \\
108 \\
134 \\
203 \\
201 \\
251 \\
201 \\
197 \\
229 \\
197\end{array}$ & $\begin{array}{l}249 \\
196 \\
235 \\
235 \\
240 \\
268 \\
253 \\
211 \\
265 \\
241\end{array}$ & $\begin{array}{r}8 \\
5 \\
27 \\
3 \\
7 \\
3 \\
9 \\
3 \\
5 \\
4\end{array}$ \\
\hline
\end{tabular}


Tab. 2. Continued.

\begin{tabular}{|c|c|c|c|c|c|c|c|c|c|c|c|c|}
\hline \multirow[b]{2}{*}{$\begin{array}{l}\text { Consti- } \\
\text { tuent }\end{array}$} & \multirow[b]{2}{*}{$\begin{array}{l}\text { Labor- } \\
\text { atoryb) }\end{array}$} & \multicolumn{5}{|c|}{ First values } & \multicolumn{4}{|c|}{ Second values } & \multirow[b]{2}{*}{ Max.f) } & \multirow[b]{2}{*}{$\begin{array}{l}\text { SD } \\
\text { within } \\
\text { series }\end{array}$} \\
\hline & & Mean & $\mathrm{SDC})$ & Mediand) & Min.e) & $\operatorname{Max} . \hat{f}$ & Mean & SDc) & Mediand) & Min.e) & & \\
\hline $\begin{array}{l}\gamma \text {-Glu- } \\
\text { tamyl- } \\
\text { transferase } \\
\text { (U/l) }\end{array}$ & $\begin{array}{l}A \\
C \\
D \\
E \\
F \\
G \\
H \\
I \\
J \\
K\end{array}$ & $\begin{array}{l}43.7 \\
48.3 \\
47.8 \\
48.3 \\
47.4 \\
48.9 \\
46.7 \\
47.7 \\
47.5 \\
46.1\end{array}$ & $\begin{array}{l}1.7 \\
1.7 \\
1.3 \\
1.0 \\
2.1 \\
0.6 \\
1.6 \\
0.5 \\
0.9 \\
0.8\end{array}$ & $\begin{array}{l}43.4 \\
48.9 \\
48.1 \\
48.4 \\
47.9 \\
48.9 \\
46.3 \\
47.7 \\
47.7 \\
46.3\end{array}$ & $\begin{array}{l}41.1 \\
43.3 \\
45.2 \\
46.8 \\
40.9 \\
47.5 \\
44.0 \\
46.7 \\
45.6 \\
45.1\end{array}$ & $\begin{array}{l}47.4 \\
50.1 \\
49.2 \\
49.8 \\
49.4 \\
49.8 \\
49.8 \\
48.4 \\
49.4 \\
47.4\end{array}$ & $\begin{array}{l}43.9 \\
49.1 \\
48.5 \\
48.4 \\
47.1 \\
48.9 \\
46.9 \\
47.7 \\
47.8 \\
45.9\end{array}$ & $\begin{array}{l}1.3 \\
1.1 \\
0.9 \\
1.0 \\
2.2 \\
0.6 \\
1.7 \\
0.6 \\
1.1 \\
0.9\end{array}$ & $\begin{array}{l}43.7 \\
49.1 \\
49.0 \\
48.5 \\
47.5 \\
48.8 \\
47.1 \\
47.7 \\
47.8 \\
46.0\end{array}$ & $\begin{array}{l}42.0 \\
47.4 \\
46.7 \\
46.3 \\
41.1 \\
47.4 \\
42.8 \\
46.5 \\
46.0 \\
44.4\end{array}$ & $\begin{array}{l}46.9 \\
51.1 \\
49.8 \\
49.8 \\
49.4 \\
49.9 \\
50.9 \\
48.8 \\
49.8 \\
47.4\end{array}$ & $\begin{array}{l}0.9 \\
1.0 \\
1.2 \\
0.4 \\
1.0 \\
0.3 \\
1.0 \\
0.4 \\
0.6 \\
0.7\end{array}$ \\
\hline
\end{tabular}

a) before the performance of any validity check

b) code of the respective laboratory

c) standard deviation (s) between series

The following calculations were performed:

The correlation coefficients $r_{k n}$ between known control and unknown sample on the one hand and $r_{b l}$ between blind control and unknown sample on the other hand, were calculated separately for each constituent and each laboratory.

First stage: First and second values (approximately 36 paired values) were pooled. Then we took only the first values of the double determinations (approximately 18 paired values). Each $r_{k n}$ and $r_{b 1}$ was tested (19) to see whether it was larger than zero (one-sided test, error probability $\alpha=5 \%$ ). Then we compared $r_{b l}$ with the corresponding $I_{k n}$ in both cases and for all laboratories and counted up how often $r_{b l}$ was larger than $r_{k n}$ and conversely how often $r_{b l}$ was smaller than $r_{k n}$. Finally we tested (19) whether the first number was significantly larger than the second one (one-sided test, $\alpha=5 \%$ ).

Second stage: Up to now all analytical data had been used. The values of the unknown sample had not been checked by means of the controls. From now on we used those series only which met the following validity criterion:

Both values of the unknown sample were accepted as valid in a series, provided both values of the known control of this series were within the uncertainty limits of the assigned value given by the manufacturer. Otherwise the whole series was eliminated.

This check was performed on all series of all laboratories and all constituents. Then we computed the standard deviation $s_{\mathrm{w}}$ within and $s_{\mathrm{b}}$ between valid series, for each laboratory and each constituent. As to $s_{b}$, the standard deviations of the first values and of the second values were evaluated separately and then pooled to $s_{\mathbf{b}}$.

Third stage: In the following we used those series only which met the following modified validity criterion:

The first value of the unknown sample was accepted as valid in a series provided the first value of the known control as well as the first value of the blind control of the series were within the uncertainty limits of the respective assigned value given by the manufacturer(s). In all other cases the whole series was eliminated.

This check was performed on all series of all laboratories and all constituents.

Results

\section{Correlations between controls and unknown sample}

Table 3 contains all correlation coefficients $r_{k n}$ between known control and unknown sample and $r_{b l}$ between blind control and unknown sample. Those
d) median of all series
e) smallest value of all series
f) largest value of all series

Tab. 3. Correlation coefficients $\mathbf{r}_{\mathbf{k}}$ between known control and unknown sample and $r_{b l}$ between blind control and unknown sample.

\begin{tabular}{|c|c|c|c|c|c|}
\hline \multirow{2}{*}{$\begin{array}{l}\text { Consti- } \\
\text { tuent }\end{array}$} & \multirow{2}{*}{$\begin{array}{l}\text { Labor- } \\
\text { atory }\end{array}$} & \multicolumn{2}{|c|}{ Using both values } & \multicolumn{2}{|c|}{$\begin{array}{l}\text { Using first values } \\
\text { only }\end{array}$} \\
\hline & & $\mathbf{r}_{\mathbf{k n}}$ & $\mathrm{r}_{\mathrm{bl}}$ & $\mathbf{r}_{\mathbf{k n}}$ & $\mathrm{r}_{\mathbf{b l}}$ \\
\hline Creatinine & $\begin{array}{l}\text { A } \\
\text { C } \\
\text { D } \\
\text { E } \\
\text { F } \\
\text { G } \\
H \\
\text { I } \\
\text { J } \\
\text { K }\end{array}$ & $\begin{array}{l}0.531^{*} \\
0.328^{*} \\
0.377^{*} \\
0.023 \\
0.107 \\
0.258 \\
0.776^{*} \\
-.032 \\
0.551^{*} \\
0.289^{*}\end{array}$ & $\begin{array}{l}0.665^{*} \\
0.520^{*} \\
0.836^{*} \\
0.162 \\
0.127 \\
0.431^{*} \\
0.812^{*} \\
-.174 \\
0.238 \\
0.691^{*}\end{array}$ & $\begin{array}{l}0.583^{*} \\
0.442^{*} \\
0.287 \\
-.072 \\
0.045 \\
0.238 \\
0.889^{*} \\
-.050 \\
0.555^{*} \\
0.177\end{array}$ & $\begin{array}{l}0.638^{*} \\
0.371 \\
0.809 * \\
0.252 \\
0.115 \\
0.359 \\
0.829 * \\
-.117 \\
0.377 \\
0.679 *\end{array}$ \\
\hline Glucose & $\begin{array}{l}A \\
C \\
D \\
E \\
F \\
G \\
H \\
I \\
J \\
K\end{array}$ & $\begin{array}{l}0.382^{*} \\
-.122 \\
0.571^{*} \\
0.325^{*} \\
0.237 \\
0.069 \\
0.572^{*} \\
-.138 \\
0.102 \\
0.122\end{array}$ & $\begin{array}{l}0.122 \\
0.170 \\
0.725^{*} \\
-.032 \\
0.507^{*} \\
0.588^{*} \\
0.659^{*} \\
0.014 \\
0.553^{*} \\
-.030\end{array}$ & $\begin{array}{l}0.362 \\
0.190 \\
0.481^{*} \\
0.443^{*} \\
0.486^{*} \\
0.152 \\
0.558^{*} \\
-.143 \\
-.088 \\
-.058\end{array}$ & $\begin{array}{l}0.473 * \\
0.445^{*} \\
0.723^{*} \\
0.124 \\
0.652^{*} \\
0.584 * \\
0.580^{*} \\
0.048 \\
0.597^{*} \\
-.236\end{array}$ \\
\hline Urea & $\begin{array}{l}\text { A } \\
\text { C } \\
\text { D } \\
\text { E } \\
\text { F } \\
\text { G } \\
\text { H } \\
\text { I } \\
\text { J } \\
\text { K }\end{array}$ & $\begin{array}{l}0.122 \\
0.079 \\
0.192 \\
0.716^{*} \\
0.332^{*} \\
0.124 \\
0.023 \\
-.140 \\
0.503^{*} \\
0.208\end{array}$ & $\begin{array}{l}0.291^{*} \\
0.093 \\
0.727^{*} \\
0.615^{*} \\
0.343^{*} \\
0.425^{*} \\
0.167 \\
-.166 \\
0.483^{*} \\
0.320^{*}\end{array}$ & $\begin{array}{l}-.089 \\
0.206 \\
0.293 \\
0.576^{*} \\
-.060 \\
0.463^{*} \\
0.367 \\
0.196 \\
0.516^{*} \\
0.297\end{array}$ & $\begin{array}{l}0.122 \\
-.222 \\
0.753 \\
0.587^{*} \\
0.496 * \\
0.346 \\
0.367 \\
-.150 \\
0.648^{*} \\
0.344\end{array}$ \\
\hline $\begin{array}{l}\text { Alanine } \\
\text { amino- } \\
\text { transferase }\end{array}$ & $\begin{array}{l}\text { A } \\
\text { B } \\
\text { C } \\
\text { D } \\
\text { E } \\
\text { F } \\
\text { G } \\
\text { H } \\
\text { I } \\
\text { J } \\
\text { K }\end{array}$ & $\begin{array}{l}0.103 \\
0.050 \\
0.215 \\
0.063 \\
0.138 \\
0.406^{*} \\
0.177 \\
0.312^{*} \\
0.407^{*} \\
0.359^{*} \\
0.389^{*}\end{array}$ & $\begin{array}{l}0.321^{*} \\
0.160 \\
0.454^{*} \\
0.589^{*} \\
0.006 \\
0.600^{*} \\
0.522^{*} \\
0.353^{*} \\
0.169 \\
0.147 \\
0.432 *\end{array}$ & $\begin{array}{l}-.184 \\
0.240 \\
0.375 \\
0.057 \\
-.004 \\
0.448^{*} \\
0.018 \\
0.032 \\
0.504^{*} \\
0.513^{*} \\
0.565^{*}\end{array}$ & $\begin{array}{l}0.268 \\
0.131 \\
0.356 \\
0.792 * \\
-.178 \\
0.581 * \\
0.579 * \\
0.299 \\
0.322 \\
0.220 \\
0.735 *\end{array}$ \\
\hline
\end{tabular}


Tab. 3. Continued.

\begin{tabular}{|c|c|c|c|c|c|}
\hline \multirow{2}{*}{$\begin{array}{l}\text { Consti- } \\
\text { tuent }\end{array}$} & \multirow{2}{*}{$\begin{array}{l}\text { Labor- } \\
\text { atory }\end{array}$} & \multicolumn{2}{|c|}{ Using both values } & \multicolumn{2}{|c|}{$\begin{array}{l}\text { Using first values } \\
\text { only }\end{array}$} \\
\hline & & $\mathbf{r}_{\mathbf{k n}}$ & $r_{\mathrm{bl}}$ & $r_{k n}$ & $r_{b 1}$ \\
\hline $\begin{array}{l}\text { Aspartate } \\
\text { amino- } \\
\text { transferase }\end{array}$ & $\begin{array}{l}\text { A } \\
\text { B } \\
\text { C } \\
\text { E } \\
\text { F } \\
\text { G } \\
\text { H } \\
\text { I } \\
\text { J } \\
\text { K } \\
\text { L }\end{array}$ & $\begin{array}{l}0.219 \\
0.500^{*} \\
0.499^{*} \\
0.137 \\
0.537^{*} \\
-.047 \\
0.392^{*} \\
-.054 \\
0.625^{*} \\
0.096 \\
0.544^{*}\end{array}$ & $\begin{array}{l}0.163 \\
0.384^{*} \\
0.301^{*} \\
0.170 \\
0.121 \\
0.024 \\
0.353^{*} \\
0.057 \\
0.511^{*} \\
0.616^{*} \\
0.561^{*}\end{array}$ & $\begin{array}{l}0.112 \\
0.609^{*} \\
0.507^{*} \\
0.194 \\
0.577^{*} \\
-.086 \\
0.434^{*} \\
0.213 \\
0.719^{*} \\
0.049 \\
0.566^{*}\end{array}$ & $\begin{array}{l}0.053 \\
0.356 \\
0.360 \\
0.340 \\
0.040 \\
0.061 \\
0.540^{*} \\
0.029 \\
0.613^{*} \\
0.801^{*} \\
0.575^{*}\end{array}$ \\
\hline $\begin{array}{l}\text { Creatine } \\
\text { kinase }\end{array}$ & $\begin{array}{l}\text { A } \\
\text { C } \\
\text { D } \\
\text { E } \\
\text { F } \\
\text { G } \\
\text { H } \\
\text { I } \\
\text { J } \\
\text { K }\end{array}$ & $\begin{array}{l}0.144 \\
0.239 \\
0.847 * \\
-.247 \\
0.169 \\
-.117 \\
0.124 \\
0.176 \\
0.160 \\
-.087\end{array}$ & $\begin{array}{l}0.350^{*} \\
0.019 \\
0.146 \\
0.211 \\
0.616^{*} \\
-.114 \\
0.169 \\
0.316^{*} \\
0.571^{*} \\
0.225\end{array}$ & $\begin{array}{l}0.268 \\
0.238 \\
0.861 * \\
-.217 \\
0.012 \\
-.057 \\
-.008 \\
-.013 \\
0.300 \\
0.097\end{array}$ & $\begin{array}{l}0.560^{*} \\
0.238 \\
0.199 \\
0.149 \\
0.685^{*} \\
-.103 \\
0.220 \\
-.123 \\
0.613^{*} \\
0.071\end{array}$ \\
\hline $\begin{array}{l}\gamma \text {-Glutamyl- } \\
\text { transferase }\end{array}$ & $\begin{array}{l}\text { A } \\
\text { C } \\
\text { D } \\
\text { E } \\
\text { F } \\
\text { G } \\
\text { H } \\
\text { I } \\
\text { J } \\
\text { K }\end{array}$ & $\begin{array}{l}0.439^{*} \\
0.349^{*} \\
0.406^{*} \\
0.332^{*} \\
0.326^{*} \\
0.174 \\
0.382^{*} \\
0.140 \\
0.303^{*} \\
0.351^{*}\end{array}$ & $\begin{array}{l}0.524^{*} \\
0.369^{*} \\
0.547^{*} \\
0.323^{*} \\
0.527^{*} \\
0.604^{*} \\
0.365^{*} \\
-.023 \\
-.089 \\
0.194\end{array}$ & $\begin{array}{l}0.299 \\
0.256 \\
0.424 * \\
0.408^{*} \\
0.133 \\
0.121 \\
0.349 \\
0.063 \\
0.256 \\
0.065\end{array}$ & $\begin{array}{l}0.420^{*} \\
0.442^{*} \\
0.561^{*} \\
0.232 \\
0.531^{*} \\
0.615^{*} \\
0.479^{*} \\
0.062 \\
-.114 \\
0.259\end{array}$ \\
\hline fotar numue & & J3 & +2 & 24 & 32 \\
\hline
\end{tabular}

All correlation coefficients which are larger than the one-sided critical value $(\alpha=5 \%)$ are starred $(*)$.

$\mathrm{r}_{\mathrm{kn}}$ and $\mathrm{r}_{\mathrm{bl}}$ significantly $(\alpha=5 \%)$ larger than zero are starred.

If these significances occurred purely at random their proportion relative to the number 72 of sets of analytical values could be expected to be about $\alpha=5 \%$. In fact, their proportion is significantly higher (19). Consequently, controls and unknown sample are found to be correlated ${ }^{3}$ ) so that it makes sense to compare $r_{k n}$ and $x_{b l}$. Table 4 contains the number of laboratories and constituents with $r_{b l} \geqslant r_{k n}$.

Comparison of these figures by means of a test (19) shows in both cases that $\mathbf{r}_{b l} \geqslant r_{k n}$ occurs significantly more frequently than $r_{b l}<r_{k n}$ (error probability $\alpha=5 \%$ ).

The philosophy of internal quality control is based on the assumption that control and unknown sample take

3) We use the correlation coefficient here as a familiar notion in order not to anticipate the results of the following paper (22). Moreover, the correlation between unknown sample and the controls was expected.
Tab. 4. Number of laboratories and constituents with $\left.{ }^{a}\right) r_{b l} \geqslant r_{k n}$.

\begin{tabular}{lll}
\hline & $\begin{array}{l}\text { Using both } \\
\text { values }\end{array}$ & $\begin{array}{l}\text { Using first } \\
\text { values only }\end{array}$ \\
\hline $\mathbf{r}_{\mathbf{b l}} \geqslant \mathbf{r}_{\mathbf{k n}}$ & 47 & 45 \\
$\mathbf{r}_{\mathbf{b l}}<\mathbf{r}_{\mathbf{k n}}$ & 25 & 27 \\
\hline Total & 72 & 72 \\
\hline
\end{tabular}

a) for explanations see table 3 .

a largely parallel course, whereas the magnitude of the analytical results of the two sera might be quite different. So it is reasonable to compute the two correlation coefficients $r_{b l}$ and $r_{k n}$ as measures of the parallel course of each; this serves to check whether the blind control or the known control better fulfills this criterion.

Since we wanted a measure for parallelity, we used the correlation coefficients independently of the distribution of the analytical values.

Actually it happened that some correlation coefficients were negative. But in this context negative correlation coefficients do not make sense. So it seemed reasonable to restrict ourselves to those laboratories and constituents with positive correlation coefficients $r_{b l}$ and $r_{k n}$. The corresponding results are given in table 5 .

Comparison of these figures by means of the test (19) again shows in both cases that $r_{b l} \geqslant r_{k n}$ occurs significantly more frequently (error probability $\alpha=5 \%$ ).

Therefore it can be concluded that the blind control is more parallel to the unknown sample, than is the known control.

Tab. 5. Number of laboratories and constituents witha) $\mathrm{r}_{\mathrm{bl}} \geqslant \mathrm{r}_{\mathrm{kn}}$ both being positive.

\begin{tabular}{lll}
\hline & Both values & $\begin{array}{l}\text { First values } \\
\text { only }\end{array}$ \\
\hline $\mathrm{r}_{\mathbf{b l}} \geqslant \mathrm{r}_{\text {kn }}$ & 41 & 36 \\
$\mathrm{r}_{\mathrm{bl}}<\mathrm{r}_{\mathrm{kn}}$ & 18 & 19 \\
\hline Total & 59 & 55 \\
\hline
\end{tabular}

a) for explanations see table 3 .

Precision within and between series (using both values and validity checks by means of the known control only)

We started from 1272 series derived from all laboratories and constituents. After the validity check based on the known control 1225 series were left. There was only one out of 72 sets of analytical values with $s_{b} \gg s_{w}$, namely one laboratory with creatine kinase, see figure 1 .

$\mathrm{s}_{\mathrm{w}}$ is a measure of precision within series. By experience, $\mathrm{s}_{\mathrm{w}}$ is indeed usually smaller than $\mathrm{s}_{\mathrm{b}}$, the precision between series. However, if $s_{b}$ exceeds $s_{w}$ essentially the analytical method is unstable from series to series so that 

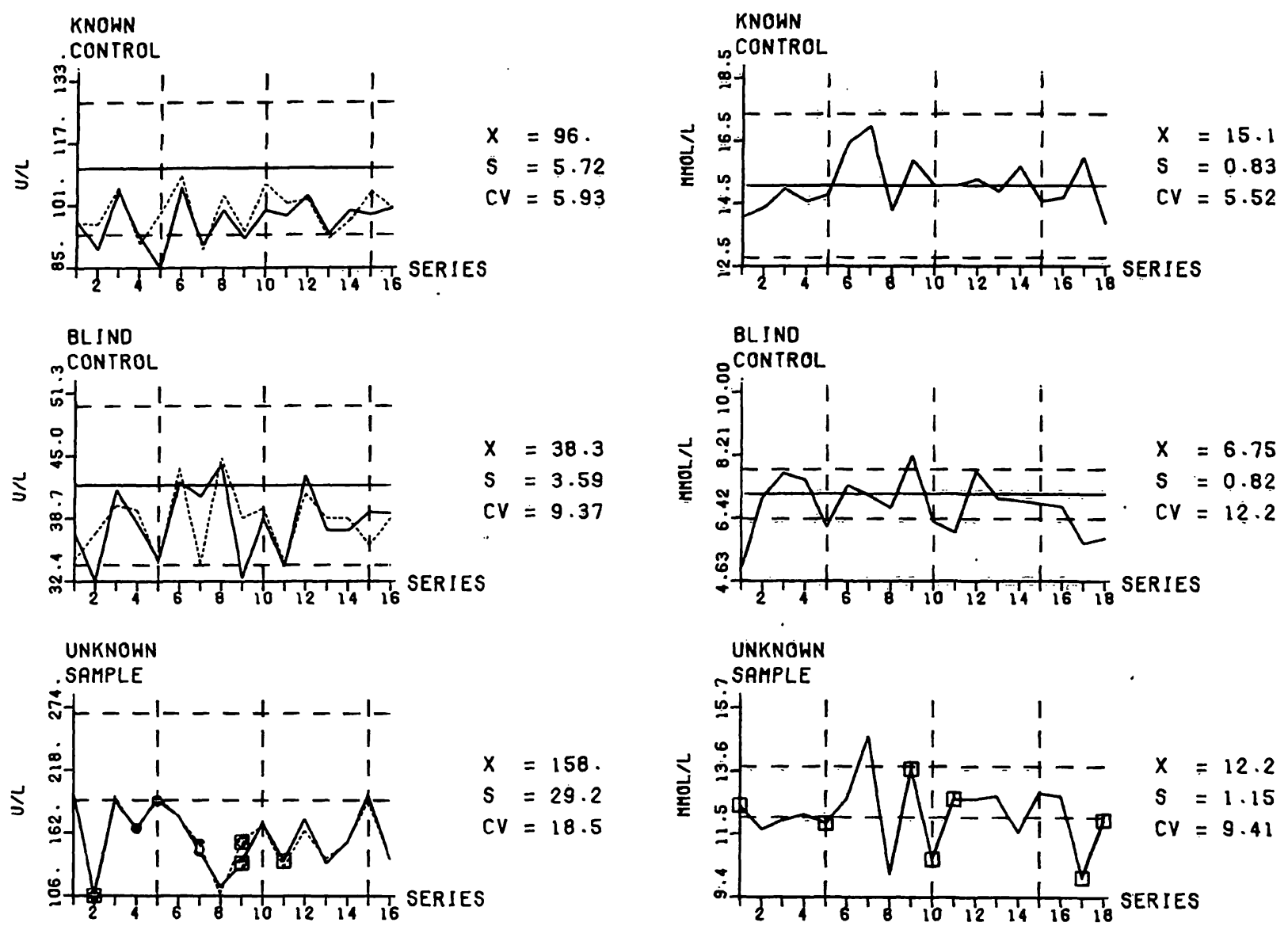

Fig. 1. Analytical results for creatine kinase from the mentioned laboratory.

Plotter printout

Ordinate: Creatine kinase [U/1].

Abscissa: Series no.

First values (solid line) and second values (dotted line) of double determinations of all three sera are plotted against the running number of series. In the controls the solid horizontal lines give the assigned values and the dashed ones their uncertainty limits. In the unknown sample however the dashed lines limit the range in which $95 \%$ of all valid values of all other laboratories lie. The vertical dashed lines facilitate the comparison of the sera. Furthermore, in the unknown sample $a \bullet(a)$ indicates that this value is eliminated by means of the known (blind) control. Mean value $x$, standard deviation $s$, and coefficient of variation $\mathrm{CV}$ of the first values of the double determinations are also given for each serum.

the analytical values are systematically incorrect. Therefore, this laboratory is eliminated from the later evaluation of creatine kinase.

Efficiency of known and blind control (using first values only and validity checks by means of both controls)

We started from 1237 series left after the validity check based on the known control only. By additional elimination based on both controls this figure was reduced to 1197 valid series. The resulting elimination proportion of $3.2 \%$ is caused by the blind control only. How-

Fig. 2. Analytical results for urea from the mentioned laboratory. Plotter printout

Ordinate: Urea [mmol/l].

Abscissa: Series no.

First values only of double determinations of all three sera are plotted against the running number of series. For the other details see figure 1 .

ever, this cannot be explained as those $5 \%$ which might statistically be expected to be eliminated when the manufacturer's $95 \%$ uncertainty interval is used. This statement is based on the fact that only about $57 \%$ of these eliminated series were uniformly distributed over all laboratories and constituents, whereas the remaining $43 \%$ were concentrated in two cases only:

Case 1: Urea, one laboratory (18 series, figure 2). The standard deviation between series is extremely high for all three sera. But the uncertainty intervals of the two control sera have different power: The uncertainty interval of the blind control is relatively smaller in comparison with the known control. Furthermore, the coefficient of variation of the blind control is more than double the value of the known control. So it is plausible that values of 7 series of the blind control, but none of the known control, are outside the limits. Therefore, all series of urea of this laboratory are eliminated.

Case 2: $\gamma$-Glutamyltransferase, one laboratory (18 series, figure 3). Similarly, the uncertainty interval of the blind control is relatively smaller than that of the known con- 

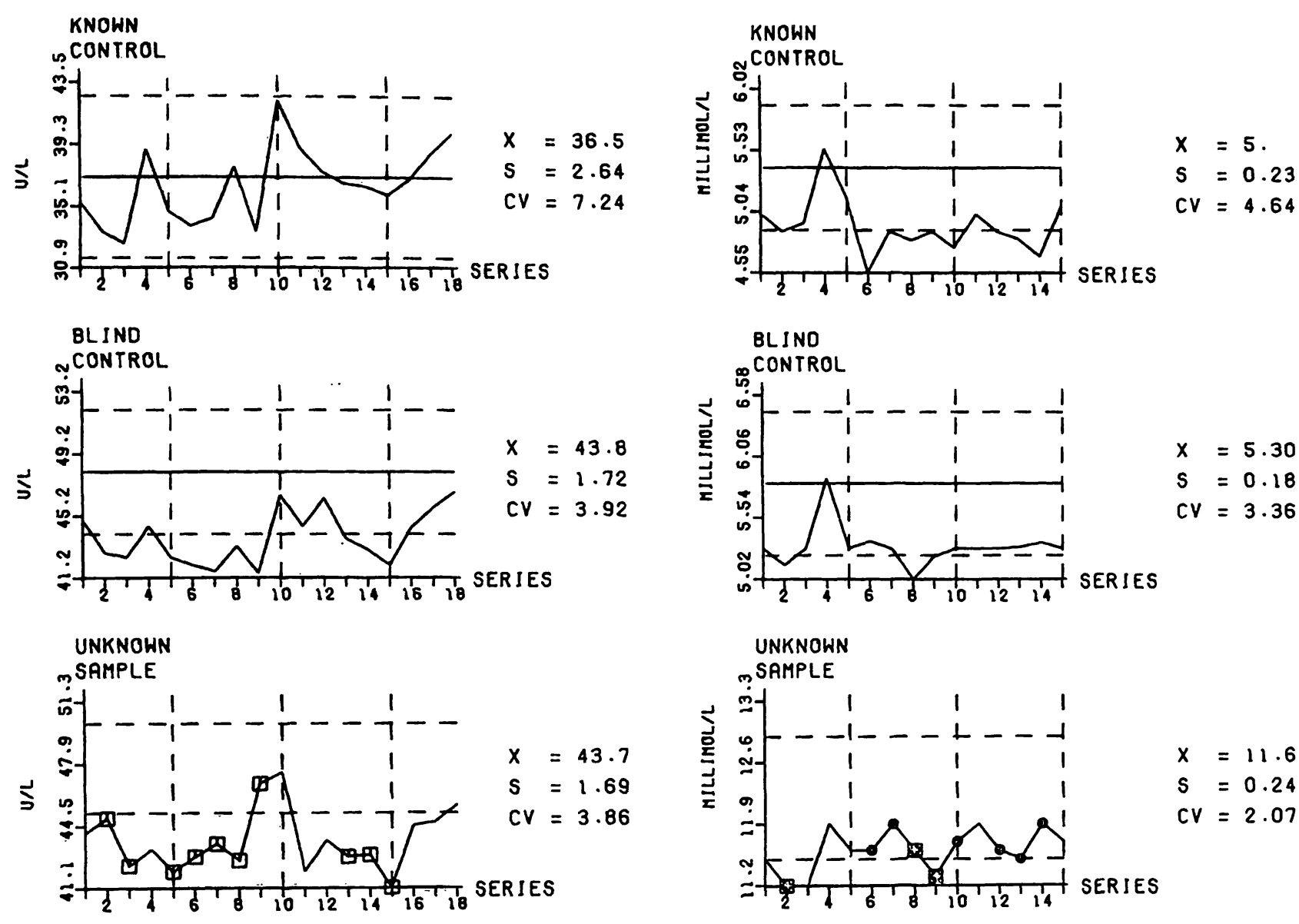

Fig. 3. Analytical results for $\gamma$-glutamyltransferase from the mentioned laboratory.

Plotter printout

Ordinate: $\gamma$-Glutamyltransferase [U/1].

Abscissa: Series no.

For details see figure 1 .

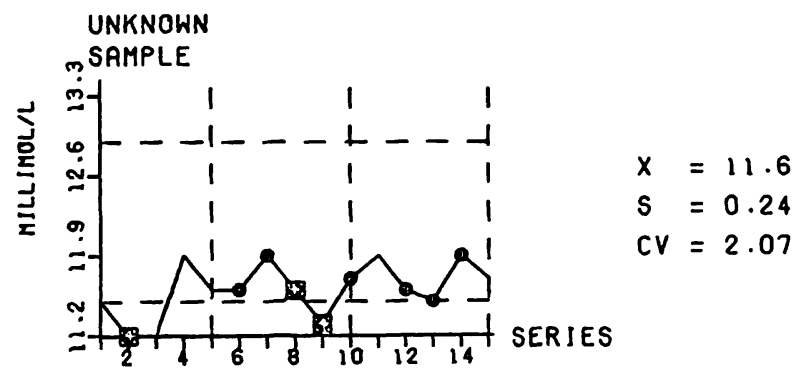

Fig. 4. Analytical results for glucose from the mentioned laboratory.

Plotter printout

Ordinate: Glucose [mmol/l]

Abscissa: Series no.

For details see figure 1.

trol and so leads to 10 eliminations. Therefore, all series of $\gamma$-glutamyltransferase of this laboratory are excluded.

In both cases the complete elimination is essential because most values of the unknown sample differ substantially from the pool of values of all other laboratories. Incorporation of the eliminated values could produce a systematically wrong assigned value.

In the above two cases the elimination is caused by the blind control only, so that the additional efficiency of this control is obvious. In two further cases the known control primarily leads to the elimination:

Case 3: Glucose, one laboratory (15 series, figure 4). Nine series are found invalid by means of the known control and three of them by means of the blind control.

others. The fact that only 6 series are found invalid is explained by different properties of the sera so that some deviations in the unknown sample are not discovered by the controls.

Another reason for exclusion is due to creatinine, one laboratory ( 17 series, figure 5). One series is eliminated by means of the known control. But all values of the unknown sample are much higher than the pool of values of the other laboratories. Therefore, all series are excluded. The reason for this failure of controls is a systematic error in performing the analyses of the unknown sample, but not of the controls.

The number of laboratories remaining after these eliminations is given in table 1 . All series of glucose of this laboratory are eliminated.

Case 4: Creatine kinase, one laboratory (16 series, figure 1). Six series are eliminated by means of both controls so that all series of creatine kinase of this laboratory are excluded. This is essential because most values of the unknown sample of this laboratory are substantially lower than the pool of values of the

\section{Discussion}

The course of a blind control correlates better with the unknown sample than does a known control. Furthermore, the effect of the blind control on the elimination of laboratories is greater than the control effect related 
to the double determination. There was only one laboratory the deviating results of which were recognized by
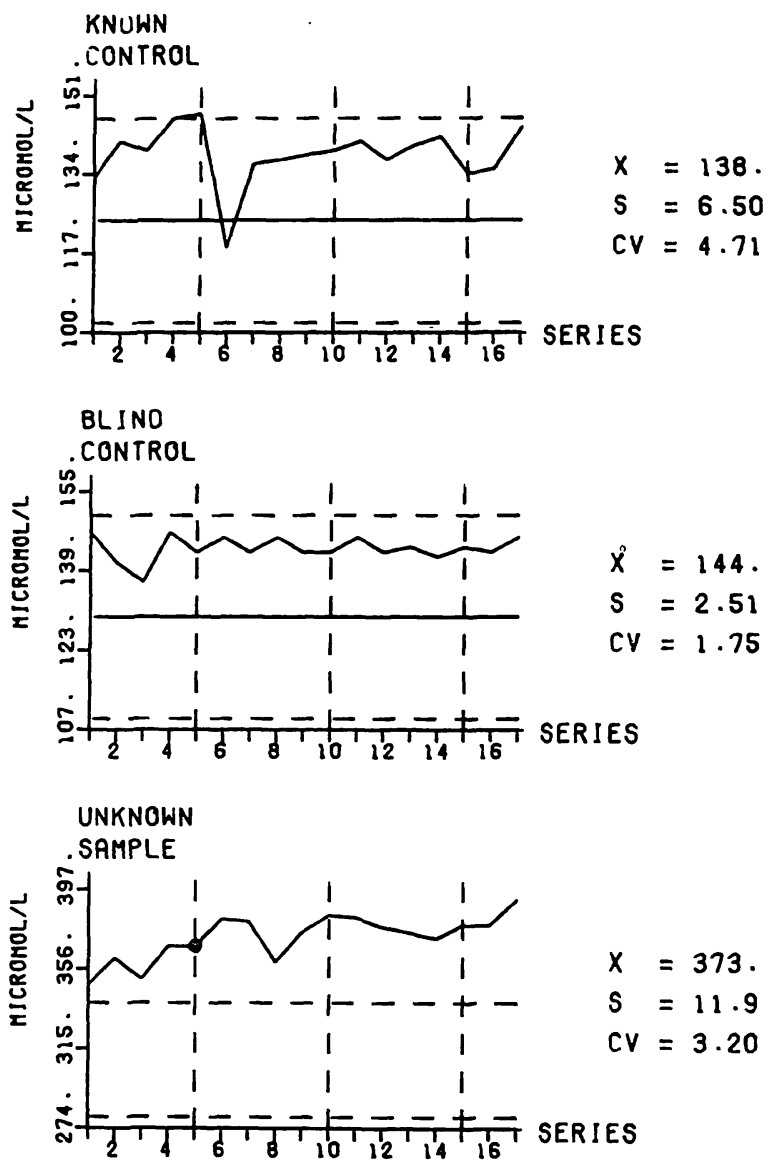

Fig. 5 Analytical results for creatinine from the mentioned laboratory.

Plotter printout

Ordinate: Creatinine [ $\mu \mathrm{mol} / 1]$

Abscissa: Series no.

For details see figure 1.

\section{References}

1. Passing, H., Glocke, M., Brettschneider, H. \& Müller, B. (1980) Lab. Med. 4, 154-159.

2. Hansert, E. \& Stamm, D. (1980) J. Clin. Chem. Clin. Biochem. $18,461-490$.

3. Schumann, V. (1976) Med. Labor. 29, 271-276.

4. The National Committee for Clinical Laboratory Standards (NCCLS), Calibration reference materials and control materials in clinical chemistry (1975).

5. Allen, J. R., Earp, R., Farrel jr., E. C. \& Gruemer, H. D. (1969) Clin. Chem. 15, 1039-1044.

6. McCormick, W., Ingelfinger, J. A., Isakson, G. \& Goldman, P. (1978) New Engl. J. Med. 299, 1118-1121.

7. Bartels, H., Boehmer, M. \& Heierli, C. (1972) Clin. Chim. Acta 37, 193-197.

8. Helger, R. R., Rindfrey, H. \& Hilgenfeldt, J. (1974).Z. Klin. Chem. Klin. Biochem. 12, 344-349.

9. Ullmann, R. \& Bonitz, K. (1976) Med. Labor, 29, 137.

10. Barthelmai, W. \& Czok, R. (1962) Klin. Wochenschr. 40, 585-589.

11. Schmidt, F. H. (1961) Klin. Wochenschr. 39, 1244-1247.

12. Slein, M. W., Cori, G. T. \& Cori, C. F. (1950) J. Biol. Chem $186,763-780$.

13. Fawcett, J. K. \& Scott, J. E. (1960) J. Clin. Pathol. 13, 156.

14. Richterich, R. (1968) Klinische Chemie, Akad. Verlagsges. Frankfurt am Main, 2nd edition, p. 254. means of both controls. Two additional laboratories however had to be eliminated on the basis of the blind control only.

The possibility of comparing precisions as described above gives the main reason for the performance of double determinations. Therefore, double determinations seem to be unneccessary. We shall confirm this conclusion in 1.c. (20) using other arguments. Rather, it is preferable to have a blind control. Both designs, either double determinations of the unknown sample only or single determination of the unknown sample as well as the addition of the blind control, result in the same analytical expenditure. For an optimal efficiency the blind control should be very similar to the unknown sample, with respect to e.g. same protein base, same degree of turbidity, lyophilisate, and so on. Clearly, the assigned values of the blind control and their uncertainty limits must be established very carefully. An analogous result is described in 1.c. (21).

The laboratories remaining after the above eliminations will be used for further evaluations in the following papers $(20,22,23)$.

\section{Acknowledgements}

This study was initiated by the Committee of Clinical Chemistry of the VDGH.

The authors thank G. von Seydlitz (Asid Bonz und Sohn GmbH), A. Berke (Boehringer Ingelheim Diagnostika GmbH), W. Bablok, $A$. Benozzi, $F$. Bosslet, M. Glocke (all Boehringer Mannheim $\mathrm{GmbH}$ ), W. von Thun (Goedecke AG), H.-G. Eisenwiener (F. Hoffmann-La Roche \& Co. AG), B. Carl (Dr. Bruno Lange GmbH), F. Schindler (F. Merck), $R$. Spaethe (Merz und Dade, Abteilung der American Hospital Supply Deutschland $\mathrm{GmbH}$ ), and D. Schlitzer (Technicon GmbH).

15. Deutsche Gesellschaft für Klinische Chemie, Standardisierung von Methoden zur Bestimmung von Enzymaktivitäten in biologischen Flüssigkeiten. Z. Klin. Chem. Klin. Biochem. 10,182 (1972).

16. Deutsche Gesellschaft für Klinische Chemie, StandardMethode zur Bestimmung der Aktivität der Creatin-Kinase. J. Clin. Chem. Clin. Biochem. 15, 249 (1977).

17. Szasz, G., Waldenstroem, J. \& Gruber, W. (1979) Clin. Chem. 25,446 .

18. Sżasz, G., Weimann, G., Stähler, F., Wahlefeld, A.-W., Persijn, J. P. (1974) Z. Klin. Chem. Klin. Biochem. 12, 228.

19. Documenta Geigy, Wissenschaftliche Tabellen, Georg Thieme Verlag, Stuttgart 1975.

20. Passing, H., Bablok, W. \& Glocke, M. (1981) J. Clin. Chem. Clin. Biochem. 19, 1167-1179.

21. Benozzi, A., Brettschneider, H., Glocke, M. \& Bernt, E. (1981) Med. Labor. in press.

22. Passing, H. (1981) J. Clin. Chem. Clin. Biochem. 19 $1145-1151$.

23. Passing, H. (1981) J. Clin. Chem. Clin. Biochem. 19 1153-1166.

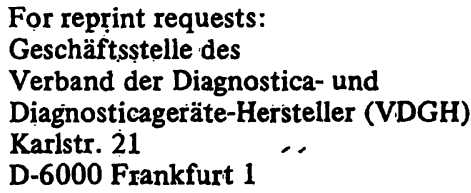

J. Clin. Chem. Clin. Biochem. / Vol. 19, 1981 / No. 11 